\title{
The WHO and why of HIV vaccine trials
}

\author{
John Moore and Roy Anderson
}

\section{A World Health Organization (WHO) advisory committee meeting in Geneva at the end of last month approved phase III trials for HIV vaccines In developing countries. What is the justification for this decision?}

WHY has a WHO committee, of which we were members, just approved HIV vaccine testing in developing countries (see Nature 371, 644; 1994) when the National Institutes of Health had earlier decided not to go ahead with trials in the United States? The approval was given for phase III trials (efficacy studies in communities where HIV is being transmitted) of recombinant subunit HIV envelope-protein vaccines which are not being undertaken in the United States because of uncertain efficacies of the available gp120 candidate immunogens. In our view, the two decisions are not inconsistent, because the circumstances influencing the desirability of a phase III trial in the United States (where HIV infection is still largely restricted to high-risk groups) and parts of the developing world (where the prevalence of infection in pregnant women attending antenatal clinics in urban centres has reached $20 \%$ or more) are quite different.

Of the 16 million people that are estimated by WHO to have acquired HIV infection, $90 \%$ live in poor communities in the developing world. Despite these chilling statistics, which highlight the urgent need for an HIV vaccine, some may interpret the WHO decision as the result of pressure by biotechnology and pharmaceutical companies who wish to experiment with products deemed unsatisfactory for use in Western countries. But this would not be a fair reaction to the WHO decision. The WHO committee was not short of information (five biotechnology companies made presentations, two of which, Biocine and Genentech, have products available for phase III trials) and contained a broad range of expert opinion, including participants from developing countries and representatives of 'atrisk' communities. There remain many scientific uncertainties, but the committee's decision was informed and objective.

\section{Decisions}

What are the uncertainties and what were the key issues that influenced the decision? The urgent need for safe and effective HIV vaccines and the desirability of conducting vaccine-efficacy trials in communities with high rates of transmission were not in question. Vaccines are a major success story of biomedical research in the twentieth century, given the global eradication of smallpox and the successful control, at least in some parts of the world, of nine major infections - tuberculosis, diphtheria, tetanus, yellow fever, pertussis, polio, measles, mumps and rubella. New recombinant vaccines for Haemophilus influenza (serotype $\mathrm{b}-\mathrm{Hib}$ ) and hepatitis $\mathrm{B}$ virus are beginning to have a very significant impact on incidence in areas where they have been used in mass campaigns. In most of these cases, however, one factor is of major importance the comparative genetic, and in particular antigenic, stability of the infectious agent in different parts of the world. Attempts to develop vaccines with high efficacy (for example protecting $80 \%$ or more of those vaccinated), have so far failed for pathogens that exhibit marked antigenic variation both within and between hosts.

In many respects the malarial parasite, Plasmodium falciparium, is a good paradigm for HIV. It exhibits great genetic (antigenic) variability, is highly pathogenic and in global terms is a chief cause of human mortality. Further, the effector mechanisms inducing acquired immunity are poorly understood, and much controversy surrounded the initiation of phase III clinical vaccine trials in the developing world with an immunogen of uncertain efficacy. The results of randomized double-blind placebo-controlled trials in Tanzania in a region of intense malaria transmission of a synthetic polypeptide candidate vaccine (SPf66) against clinical malaria have now been published (P. L. Alonso et al. Lancet 344, $1175-1181 ; 1994)$. The study confirmed that SPf66 is safe and immunogenic but, more important, it revealed an efficacy of $31 \%$ (confidence interval $0-52 \%$ ) in 274 children aged $1-5$ years who received three doses of vaccine. Given this level of efficacy, the potential of the SPf66 vaccine as a public-health measure in Africa will be much debated in the coming months. However, as falciparum malaria causes between 1 and 3 million deaths per year, largely in young children and pregnant women in sub-saharan Africa, a vaccine with a moderate-to-low efficacy is, without doubt, a valuable addition to existing control measures:

The debate about the HIV vaccine trials among the WHO committee members centred on several issues, including the likelihood that the immunogens might enhance disease progression and perhaps infectiousness in recipients who subsequently became infected, and that they could increase the rate of HIV transmis- sion by inducing a feeling of safety in recipients, resulting in increased high-risk sexual behaviour. The latter argument is important, and careful counselling will be essential in any clinical trial, but concern over behaviour change has not prohibited the use of chemotherapeutic agents that do not eliminate HIV but prolong infectious lifespan in those treated.

The greatest concern of members of the WHO committee, however, was that the products simply would not work, in the sense that the degree of protection they conferred to an individual would be so slight as to be insignificant on a population basis. This view tended to be held by researchers who had studied the immune response to subunit vaccines in humans and animal models, whereas clinical scientists and epidemiologists were generally less swayed by uncertainties over which immunological markers best correlated with protection, or disagreements about the relevance of results from HIV-1 infection in chimpanzees to infection and disease in humans. Clinicians and epidemiologists argued that the only way to determine efficacy is to run an efficacy trial, which is both true and a truism, and that failure is an acceptable outcome. Others disagreed, and felt that the chances and consequences of failure are sufficiently great that a phase III trial of the recombinant gp120 candidates is unwarranted.

Herein lies the crux of the debate: the likelihood of the products being efficacious to any extent. Unfortunately, nobody knows with any certainty, and it all boils down to a matter of opinion. Nobody, not even the most passionate advocate of a trial, believes that efficacy would approach $100 \%$, and few at the meeting were prepared to guess at even $50 \%$. A significant minority felt that efficacy would be close to $0 \%$. They therefore argued that the available recombinant gp120 products were not suitable for a phase III trial - but they could not be certain that their $0 \%$ estimate was right. Thus, opinion was strongly divided about the efficacy of the vaccine candidates, leading to many modifications to the final statement released by WHO before all members of the committee could endorse it.

A key point in the discussion was whether a low-efficacy vaccine would be of benefit to countries where HIV is spreading rapidly both in high-risk groups and in the general sexually active popula- 
tion. It is difficult to answer this question with precision, given the many uncertainties that surround the properties of the immunogens and the factors that control transmission in a given community. Mathematical models of the transmission dynamics of HIV provide a crude template with which to explore this issue. Analyses presented at the meeting suggested that even efficacies of 30 to $50 \%$, with a 5-year protection following vaccination, could save many lives in communities in which transmission rates are high. Here lies the analogy with the recent malaria vaccine trial referred to above, in which efficacy was found to be $31 \%$. But achieving this degree of protection with an HIV immunogen may be very difficult, given the enormous genetic diversity of the virus and its continued, rapid evolution as it spreads locally and worldwide.

A further issue of relevance concerns the cost of a product to be used on a community-wide basis. Cost is a central question for all mass-immunization programmes, particularly for those in developing countries, which have limited resources for health care. At present, little is known about the likely cost of an HIV vaccine, but this issue will become of paramount importance if efficacy is low and duration of protection short. Preliminary studies based on mathematical models of HIV transmission suggest that the most beneficial vaccination programme design, in terms of minimizing the cumulative number of cases of infection in areas of intense transmission, is blanket coverage of the sexually active population, repeated annually in an unselective manner (irrespective of a previous history of vaccination). This, combined with the need to monitor continually the most appropriate immunogen(s) on which to base the vaccine due to the continuing evolution of the virus, suggests that costs of any long-term immunization programme will be high. Similar issues pertain for the new malaria vaccine.

\section{Recommendations}

So what exactly was recommended by the WHO committee? Crucially, it concluded that any decision to go ahead with a trial of any product must be made by the government of the country hosting a trial, and that phase I/II safety trials in that country should precede a phase III trial. This will, of course, expose various governments to extensive lobbying over the next few months from vaccine manufacturers and clinical-trial coordinators. But the governments concerned will have the full support of the WHO when they make a decision that is most suitable for their populations, taking into account the benefits and chances of success, and the consequences of failure.

The WHO committee stated that the particular HIV-1 subtypes circulating in the proposed trial population should be a major consideration; it was felt, for example, that a vaccine based on a subtype B envelope should not be tested in an African country where viruses of subtypes A and $\mathrm{D}$ predominate. Counselling of the trial populations as to the potential risks of vaccination and the necessity of following safe sexual practices was agreed to be mandatory; indeed, an increase in the amount of information provided to a trial population was considered by some committee members likely to have a greater impact on HIV transmission than the vaccines themselves.

This particular concern highlights a further problem - the design of a phase III trial. The numbers of people required in each arm of the trial (treated and placebo) is related both to expected efficacy of the vaccine and to the rate of HIV infection in the trial community. The latter parameter changes over time in the absence of any intervention, moving from low to high to low again as the epidemic develops. The former is difficult to predict. Both factors mitigate toward trials involving many thousands of treated individuals (perhaps tens of thousands).

Most eyes are now unofficially focused on Thailand, where the characteristics of the current HIV-1 epidemics are widely thought to be favourable for a phase III efficacy trial. Two genetically and antigenically quite divergent subtypes of HIV-1 are present in different Thai subpopulations: subtype $\mathrm{E}$ is transmitted predominantly by heterosexual contacts in northern Thailand; whereas subtypes B and $\mathrm{E}$ both circulate among injecting drug users in Bangkok. However, many of the subtype B viruses found in Thailand are somewhat divergent from the $\mathrm{MN}$ and SF-2 North American strains used to make the current gp120 subunit vaccines.

The above cohorts are the potential targets for separate efficacy trials, each of which has advantages and disadvantages. The favoured test population for the presently available subtype $B$ vaccines is the Bangkok drug users, to address the issues of inter- and intra-subtype efficacy simultaneously. However, preliminary results presented earlier this month by Marc Girard and Patricia Fultz at an AIDS vaccine conference in Washington $\mathrm{DC}$, showed that a chimpanzee infected with a subtype B virus was easily superinfected on challenge with a subtype $E$ virus. Although the WHO committee had significant reservations about the chimpanzee model, it is hard to imagine that a vaccineinduced response to a subtype B gp120 would be superior to the infectioninduced response to a subtype B virus in protecting a chimpanzee or human from infection with a subtype $E$ virus.

A second concern about a trial in the drug-user cohort is that the success or failure of a gp120 vaccine against virus transmission via injected blood may not necessarily predict success or failure of a similar vaccine in a population where transmission is predominantly sexual - in other words, in the vast majority of the cases of HIV-1 transmission worldwide. Thus, a trial in a drug-user cohort might need to be repeated in a sexualtransmission cohort, whatever its outcome.

A potentially smaller and simpler trial would involve a subtype E gp120 vaccine in the northern Thailand sexual transmission cohort. This trial might not address inter-subtype protection, but this would only be relevant if there were any significant intra-subtype efficacy, which is a far from certain outcome of any trial. Although no subtype E gp120 is yet available for an efficacy trial, there are apparently plans to make such a protein ready in a year or so. Although the WHO committee made no specific recommendations, we believe that if an efficacy trial is done at all, the best option is the simplest one: a subtype E gp120 trial in the cohort where strains of predominantly subtype $E$ are transmitted sexually.

One of the most important statements made by the WHO committee was to emphasize a long-term commitment to AIDS vaccine development and testing worldwide. All of us, government and community representatives, academic and corporate scientists, welcome this support for something to which we are all deeply committed: the eventual development of a globally effective AIDS vaccine or vaccines. The present generation of gp120 subunit vaccines was designed around 1984; in 1994 there is a clear need for better products given the expectation of low efficacy in phase III trials with the currently available vaccines. But the desire for a vaccine is universal, and the need for more national and international support for further fundamental research on the relevant topics has been clearly demonstrated.

The eyes of the world are on HIV vaccine researchers, today more than ever. The results of the development and the recent trials of the malarial vaccine hold many important lessons for the HIV research community. A consensus has been reached by the WHO in good faith: to leave a decision for a phase III trial of HIV subunit vaccines to national governments, and to support those who choose to do so. These governments are quite capable of deciding in their own best interests, so perhaps it is time for us all to leave them to do so.

John Moore is at the Aaron Diamond AIDS Research Center, New York, New York 10016, USA; Roy Anderson is in the Centre for the Epidemiology of Infectious Diseases, University of Oxford, Oxford OX1 3PS, UK. 